\title{
THE RELATIONSHIP OF THE CLINICAL RESULTS OF THE PATIENTS UNDERGOING TRANSFORAMINAL EPIDURAL INJECTION WITH PREOPERATIVE MAGNETIC RESONANCE IMAGING FINDINGS
}

\author{
๑ Bilal Aykaç, @ Abdullah Küçükalp \\ Private Hayat Hospital, Clinic of Orthopedics and Traumatology, Bursa, Turkey
}

Objective: Lumbar disc herniation (LDH) is a common cause of low back pain and lumbar radiculopathy. In this study, the relationship between clinical results and pre-procedural magnetic resonance imaging (MRI) findings of patients with LDH-related radiculopathy symptoms who underwent lumbar transforaminal epidural steroid injection (TFESI) was evaluated.

Materials and Methods: Between 2017 and 2021,65 patients who were diagnosed as having LDH clinically and radiologically and underwent new MRI examination at the latest 3 months before the procedure were included in the study. In the operating room, under the scopy imaging, $1 \mathrm{cc}$ opaque substance (iohexol) was diluted with $5 \mathrm{cc}$ isotonic solution and 1.5-2 cc of this was injected into the area for confirmation in the scopy vision. Then $1 \mathrm{cc}$ betamethasone and 4 cc $2 \%$ prilocaine hydrochloride were mixed, and 5 cc of this was injected. Pain scoring was evaluated with visual analog scale (VAS) and disability was evaluated with oswestry disability index (ODI). Pre-procedural MRIs were examined and grouped according to Michigan State University classification.

Results: The patients' median VAS and ODI scores were 8 [interquartile range (IQR): 7-8] and 74 (IQR: 67-77) before treatment; 2 (IQR: 1-3) and 14 (IQR: 10-29) in the $2^{\text {nd }}$ week; and 2 (IQR: 1-4) and 16 (IQR: 4-40) in the $3^{\text {rd }}$ month, respectively. It was determined that there was a statistically significant change in the pain and disability levels of the patients over time (ANOVA type test statistics=338,743, degree of freedom $=1,542, p$-value $<0.001$ ). It was determined that the change observed in VAS and ODI scores over time did not show a statistically significant difference according to disc type and location.

Conclusion: The TFESI is a treatment method that can be used safely, independent of the disc type and localization in the MRI performed before the procedure.

Keywords: Transforaminal injection, disc hernia, radiculopathy

\section{INTRODUCTION}

Although lumbar disc herniation (LDH) is a common cause of low back pain and lumbar radiculopathy, it also causes socioeconomic losses in society. The incidence of symptomatic LDH has been reported as $1-3 \%^{(1,2)}$. LDH welded in selected cases for radicular pain, lumbar transforaminal epidural steroid injections (TFESI) has been shown to be an effective treatment method(3). The complaints of radiculopathy and related leg pain occur together with low back pain associated with the compression of the nerve roots ${ }^{(1)}$.

There are studies indicating that TFESI is significantly effective and safe for discogenic low back pain and moderately effective in spinal stenosis ${ }^{(4)}$. Radiculopathy pain caused by lumbar disc hernia of patients can be controlled with TFESI technique, which is one of the current treatment options in patients with LDH.
Prior to the TFESI procedure, patients should have undergone medical treatment and/or physical therapy protocols. Since it is an interventional procedure; it is applied to groups of patients who cannot obtain results from non-invasive treatments(5).

The size, localization and nerve compression of the disc herniation can be seen on magnetic resonance imaging (MRI), and it also guides the clinic and treatment ${ }^{(6,7)}$. Abnormal findings on imaging can be measured more objectively. Although a standard classification for LDH cannot be fully established in $\mathrm{MRI}^{(1)}$, the Michigan State University (MSU) classification is frequently used in surgical selection as an objective measure of LDH in MRI with $98 \%$ inter-inspector reliability ${ }^{(8)}$.

The aim of this study is to examine the relationship between the clinical results of patients with radiculopathy symptoms due to LDH and who underwent TFESI with pre-procedural MRI findings. 


\section{MATERIALS AND METHODS}

\section{Study Group}

Ethics committee approval was obtained for this study from Uludağ University Faculty of Medicine Clinical Research Ethics Committee (dated 16.06.2021 and numbered 2011KAEK-26). All of the patients were selected from the patient groups who had previously received medical treatment and/ or physical therapy protocol treatment, but did not have a clinical response. Patients with a history of previous surgery, stenosis of degenerative background, surgical indication, bleeding diathesis, morbidly obese (body mass index over 40), local skin lesion and patients under 18 years of age were excluded from the study. Sixty-five patients with radiculopathy symptoms due to LDH, without acute neurological symptoms and motor loss, who were confirmed by clinical and radiological diagnosis between 2017 and 2021, and who underwent new MRI examination at the latest 3 months before the procedure, were included in the study (Figure 1).

\section{Process Preparation and Technique}

Informed consent forms were obtained from all patients before the TFESI procedure. To the patients; level detection was performed in the operating room, on the surgical table, with monitoring, in the prone position, under fluoroscopy control. After the application of $2 \%$ prilocaine hydrochloride as $5 \mathrm{cc}$ local anesthetic, the area to be injected is reached with a 22 gauge spinal needle, again under fluoroscopy control, with the posterolateral transforaminal area accompanied by anteroposterior and lateral fluoroscopy images (Figure 2), 1 cc opaque substance (iohexol), $5 \mathrm{cc}$ isotonic solution was diluted and injected into the area in a volume of 1.5-2 cc for confirmation in scopy vision (Figure 3). After the foramen and root level were determined, 1 cc betamethasone and 4 cc $2 \%$ prilocaine hydrochloride were mixed and 5 cc was applied. Patients were monitored during the procedure, while staying in contact with the patient during the procedure, whether there was severe leg pain and motor deficit with active foot movement. After the procedure, the patients were followed for at least 3 hours, and after the motor-sensory block was completely over, they were

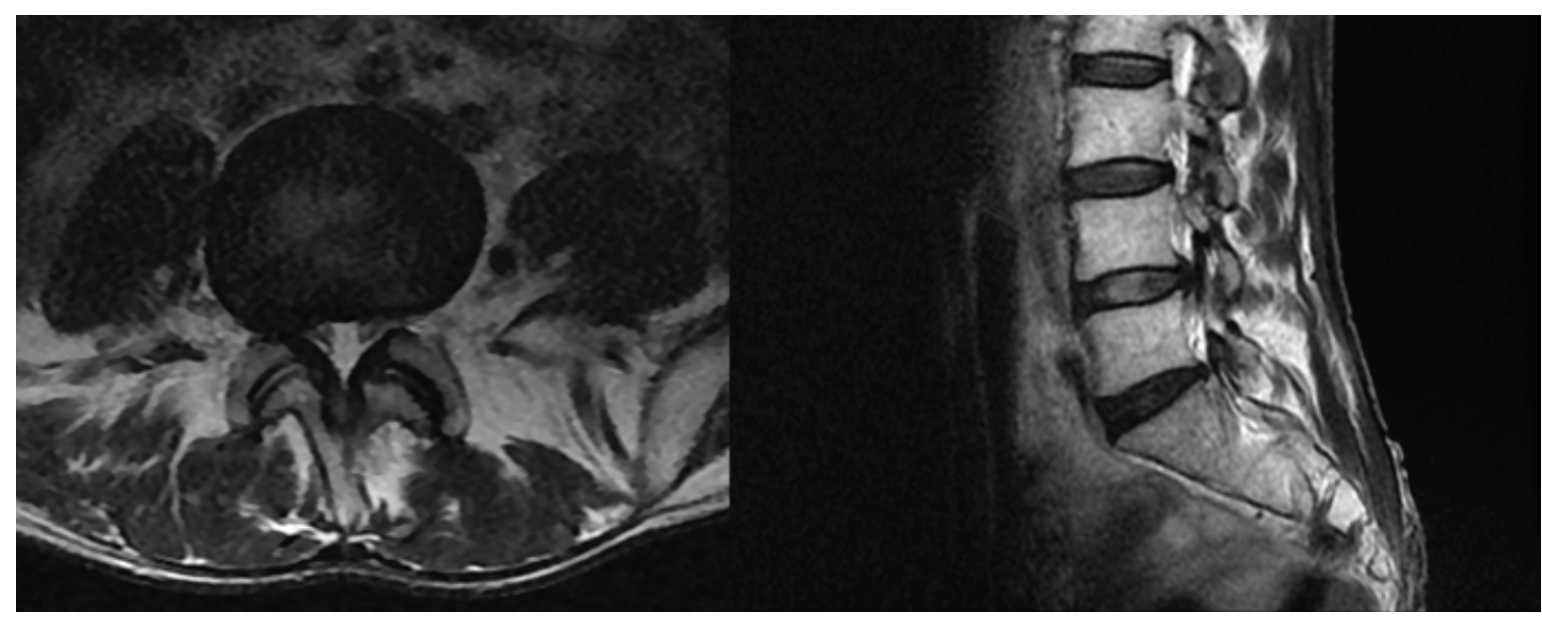

Figure 1. MRI of lumbar spine before transforaminal injection

MRI: Magnetic resonance imaging

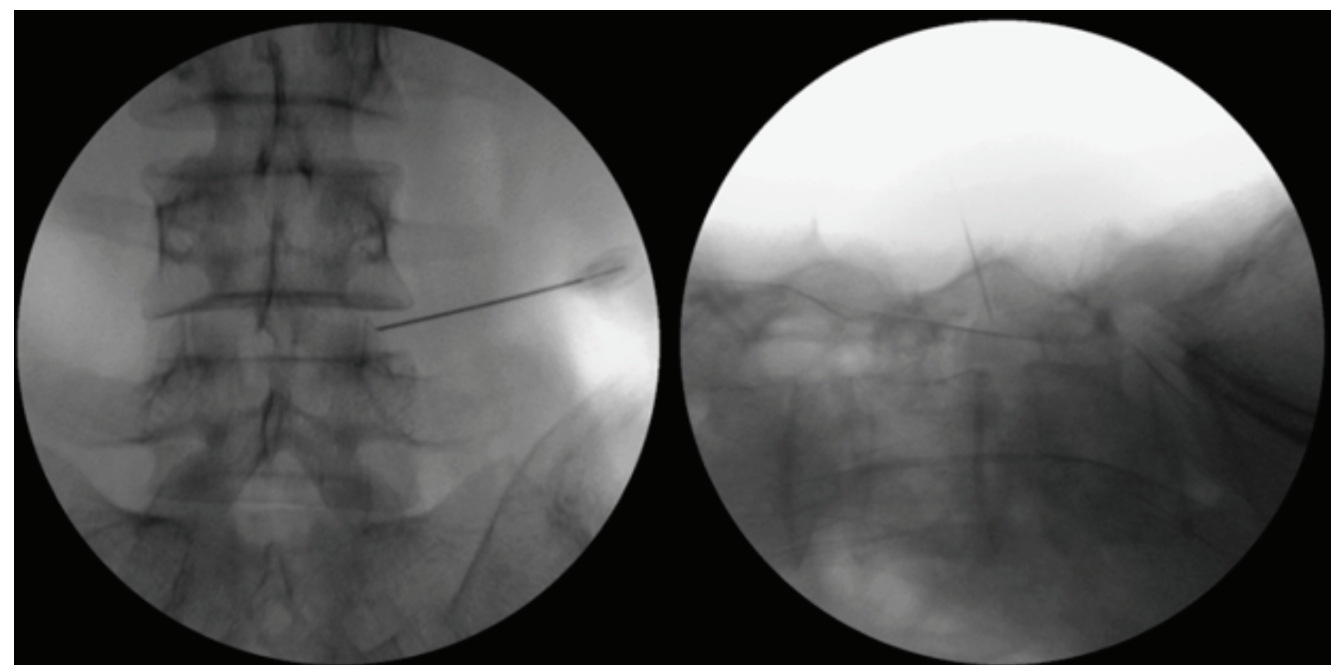

Figure 2. The antero-posterior and lateral fluoroscopic image of lumbar spine 
turkishspine

mobilized and externated. Pain scores [visual analog scale (VAS)] and functional oswestry disability index (ODI) scores were analyzed from the files of the patients who underwent TFESI procedure, before the procedure, at the $2^{\text {nd }}$ week and $3^{\text {rd }}$ month after the procedure. By examining their MRIs retrospectively; based on the MSU classification, protusion and bulging were grouped according to the herniation type, and central, posterolateral and foraminal according to their localization.

\section{Statistical Analysis}

The distributions of age, VAS and ODI were examined by using Shapiro-Wilk's tests, normality plots and skewness/kurtosis statistics. Since only age was distributed normally, it was summarized by mean \pm standard deviation while numeric rating scale and ODI were provided by median (IQR: $1^{\text {st }}$ quartile- $3^{\text {rd }}$ quartile). Frequencies (\%) were given for gender, disc type and disc localization.

The changes in VAS and ODI measurements across time was examined by LD-F1 design in overall and were compared by F1-LD-F1 design with respect to the disc type and disc localization. ANOVA type test statistics (ATS), degree of freedom (df) and $p$-values were reported for the overall time effect and group*time interaction (GTI) effects. Relative treatment effects (RTEs) were provided with $95 \%$ confidence interval by graphs. A $p$-value $<0.05$ was considered as statistically significant. Descriptive statistics were calculated by IBM SPSS Statistics 22.0 (IBM Corp. Released 2013. IBM SPSS Statistics for Windows, Version 22.0. Armonk, NY: IBM Corp.). The LD-F1 and F1-LD-F1 designs were performed using the RStudio Software program (v.1.4.1106) ${ }^{(9)}$ and the nparLD package ${ }^{(10)}$ in the R v.4.1 programming language ${ }^{(11)}$.

\section{RESULTS}

The mean age of the patients included in the study was $49.72 \pm 15.35$ years (minimum-maximum: $21-80$ ). Disc type was protruded in $50.8 \%$ ( $n=33$ ) of the patients and bulging in $49.2 \%$ $(\mathrm{n}=32)$. Disc localization was determined as posterolateral in $56.9 \%(n=37)$, central in $24.6 \%(n=16)$, and foramen/PL in $18.5 \%$ $(n=12)$.

The patients' median VAS and ODI were 8 (IQR: 7-8) and 74 (IQR: 67-77) before treatment, respectively; 2 (IQR: 1-3) and 14 (IQR: 10-29) at $2^{\text {nd }}$ week; They were 2 (IQR: 1-4) and 16 (IQR: 4-40) at $3^{\text {rd }}$ months (Table 1). It was determined that there was a statistically significant change in the pain and disability levels of the patients over time (ATS=338,743, $d f=1,542$, $p$-value $<0,001)$. When RTEs were examined, it was observed that there was a significant decrease in the $2^{\text {nd }}$ week and the pain and disability level in the $2^{\text {nd }}$ week was maintained at the $3^{\text {rd }}$ month (Figure 4).

When the pain and disability levels of the patients were analyzed by disc type, the median VAS was 8 (IQR: 7-8) before treatment and 2 (IQR: 1-3) at week 2 for both disc types. The median VAS at 3 months was 2 (IQR: 1-4) for patients with protrusion disc and 2 (IQR: $0-4$ ) for patients with bulging disc (Table 1). It was determined that the change observed in pain level over time did not show a statistically significant difference

Table 1. Patients' pain and disability levels through time with respect to the disc type

\begin{tabular}{|c|c|c|c|}
\hline & \multicolumn{3}{|c|}{ Disc Type } \\
\hline & $\begin{array}{l}\text { Overall } \\
{[n=65]}\end{array}$ & $\begin{array}{l}\text { Protrusion } \\
{[n=33]}\end{array}$ & $\begin{array}{l}\text { Bulging } \\
{[n=32]}\end{array}$ \\
\hline \multicolumn{4}{|c|}{ NRS [median (IQR)] } \\
\hline Baseline & $8(7-8)$ & $8(7-8)$ & $8(7-8)$ \\
\hline $2^{\text {nd }}$ week & $2(1-3)$ & $2(1-3)$ & $2(1-3)$ \\
\hline $3^{\text {rd }}$ month & $2(1-4)$ & $2(1-4)$ & $2(0-4)$ \\
\hline \multicolumn{4}{|c|}{ ODI [median (IQR)] } \\
\hline Baseline & $74(67-77)$ & 77 (67-79) & $70(67-77)$ \\
\hline $2^{\text {nd }}$ week & $14(10-29)$ & $14(11-28)$ & $12(9-30)$ \\
\hline $3^{\text {rd }}$ month & $16(4-40)$ & $18(4-40)$ & $12(3-38)$ \\
\hline
\end{tabular}

NRS: Numeric rating scale for pain, ODI: Oswestry disability index IQR (interquartile range): $1^{\text {st }}$ quantile- $3^{\text {rd }}$ quantile

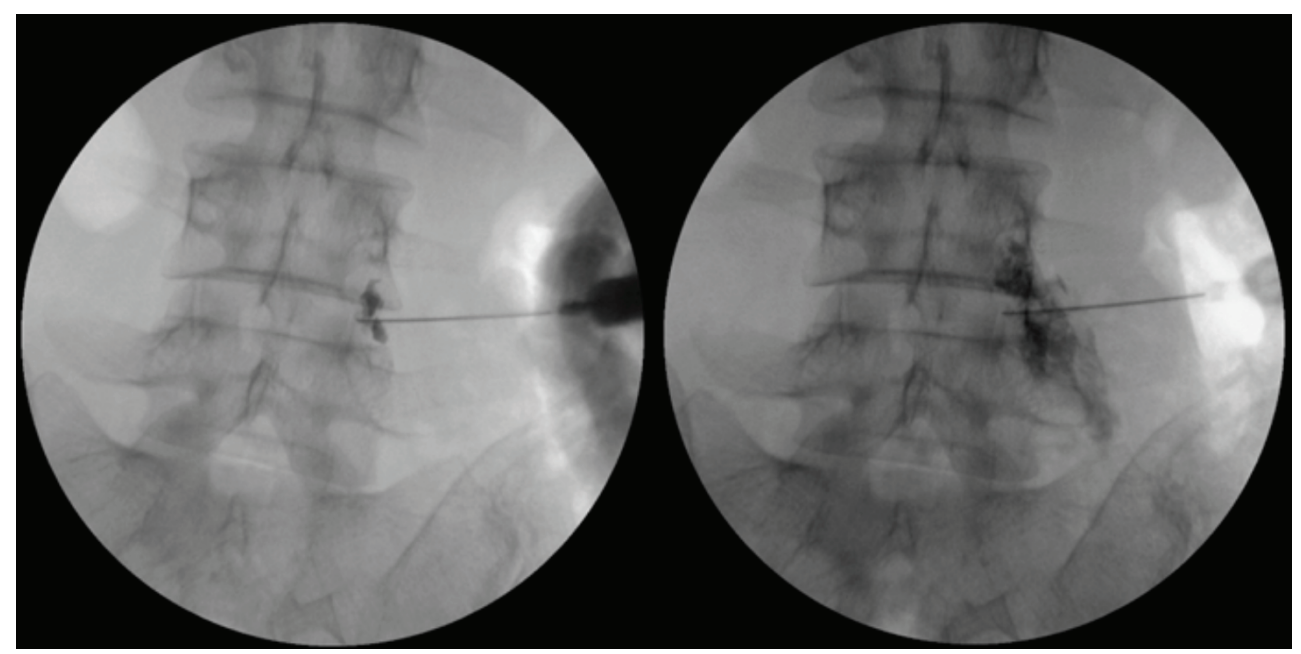

Figure 3. The fluoroscopic image of lumbar spine at the time of injection 
according to disc type ATS=0.234, df=1,536, $\mathrm{p}$-value $=0.731$ for GTI (Figure 5). The median ODI was 77 (IQR: 67-79) for patients with protrusion discs before treatment and 70 (IQR: 67-77) for patients with bulging discs; 14 (IQR: 11-28) for patients with protrusion discs at $2^{\text {nd }}$ week, 12 (IQR: 9-30) for patients with bulging discs, 18 (IQR: 4-40) for patients with protrusion discs at $3^{\text {rd }}$ month, 12 for patients with bulging discs (IOR:338) (Table 1). The change observed in ODI measurements over time did not differ according to disc type (ATS=0.279, df=1,439, $p$-value $=0.682$ for GTI, Figure 5).

The distribution of VAS and ODI measurements according to the disc location in the patients is given in Table 2. In these measurements, it was determined that the change observed over time did not show a statistically significant difference according to the disc location (ATS=1,312, df=2,722, $p$-value $=0.269$ for GTI effect in VAS; ATS $=1.332$, df $=2,555$, $p$-value $=0.264$ for GTI effect in ODI; Figure 6).

\section{DISCUSSION}

Although a standard classification for LDH in MRI cannot be fully established ${ }^{(1)}$, Mysliwiec et al. ${ }^{(8)}$ they used the MSU classification to follow the surgical route with $98 \%$ reliability between examiners, and we grouped the disc size and localization MRIs retrospectively on the basis of the MSU classification in our study. According to this; it was classified

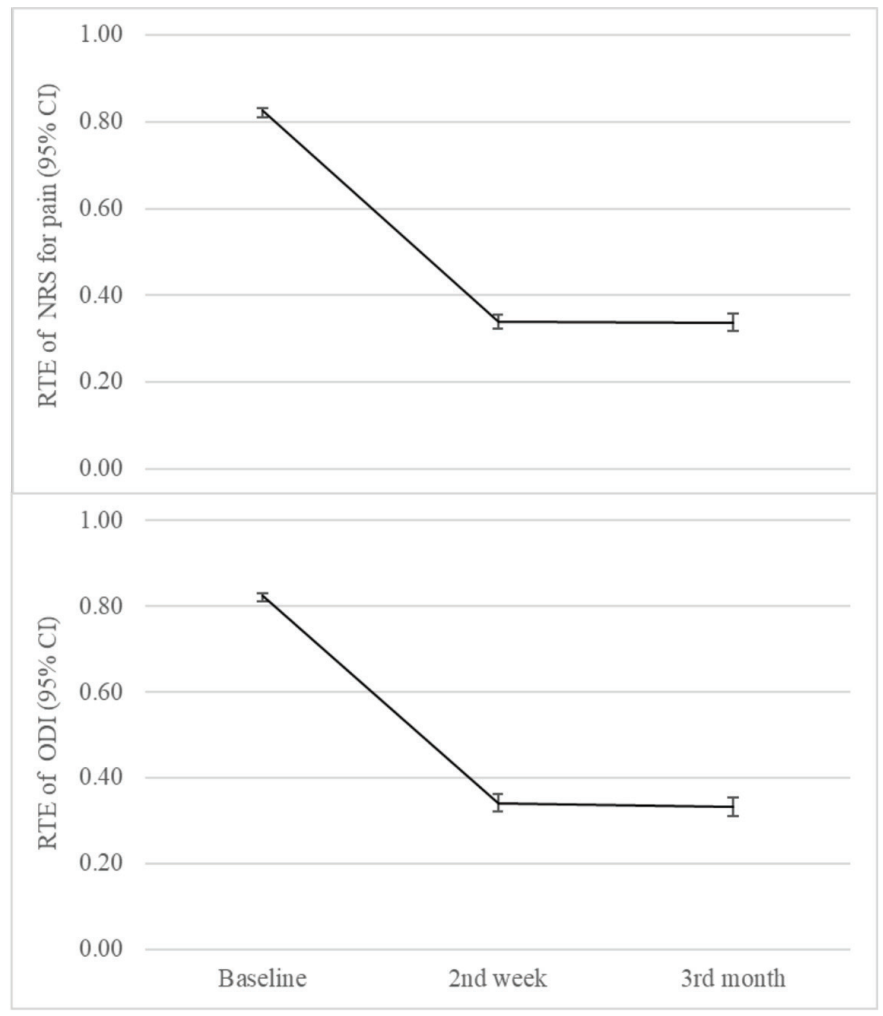

Figure 4. Overall relative treatment effect for pain and disability level

NRS: Numeric rating scale for pain, ODI: Oswestry disability index, RTE: Relative treatment effects, $\mathrm{Cl}$ : Confidence interval as bulging and protrusion based on size and shape, and as central, posterolateral and posterolateral/foraminal in terms of localization. Since there is no criterion showing disc degeneration in the current classification, soft or hard disc types were not differentiated and these criteria were not included in the study.

Manchikanti et al.(12) in his review; between 1966 and 2011, 70 publications were reviewed and the level of evidence for radiculitis secondary to disc herniation was good when

Table 2. Patients' pain and disability levels through time with respect to the disc localization

\begin{tabular}{llll}
\hline \multicolumn{5}{c}{$\begin{array}{l}\text { Disc Localization } \\
{[\mathrm{n}=12]}\end{array}$} & $\begin{array}{l}\text { Posterolateral } \\
{[\mathrm{n}=37]}\end{array}$ & $\begin{array}{l}\text { Central } \\
{[\mathrm{n}=16]}\end{array}$ \\
\hline NRS [median (IQR)] & & $8(7-8)$ \\
\hline Baseline & $8(8-8)$ & $8(7-8)$ & $2(1-4)$ \\
\hline $2^{\text {nd }}$ week & $3(2-4)$ & $2(1-3)$ & $3(1-5)$ \\
\hline $3^{\text {rd }}$ month & $3(1-5)$ & $1(0-4)$ & \\
\hline ODI [median (IQR)] & & $70(66-78)$ \\
\hline Baseline & $77(69-77)$ & $74(67-78)$ & $14(10-30)$ \\
\hline $2^{\text {nd }}$ week & $17(12-32)$ & $14(7-29)$ & $21(6-43)$ \\
\hline $3^{\text {rd }}$ month & $20(9-48)$ & $10(2-31)$ & \\
\hline
\end{tabular}

PL: Posterolateral, NRS: Numeric rating scale for pain, ODI: Oswestry disability index

IQR (interquartile range): $1^{\text {st }}$ quantile- $3^{\text {rd }}$ quantile

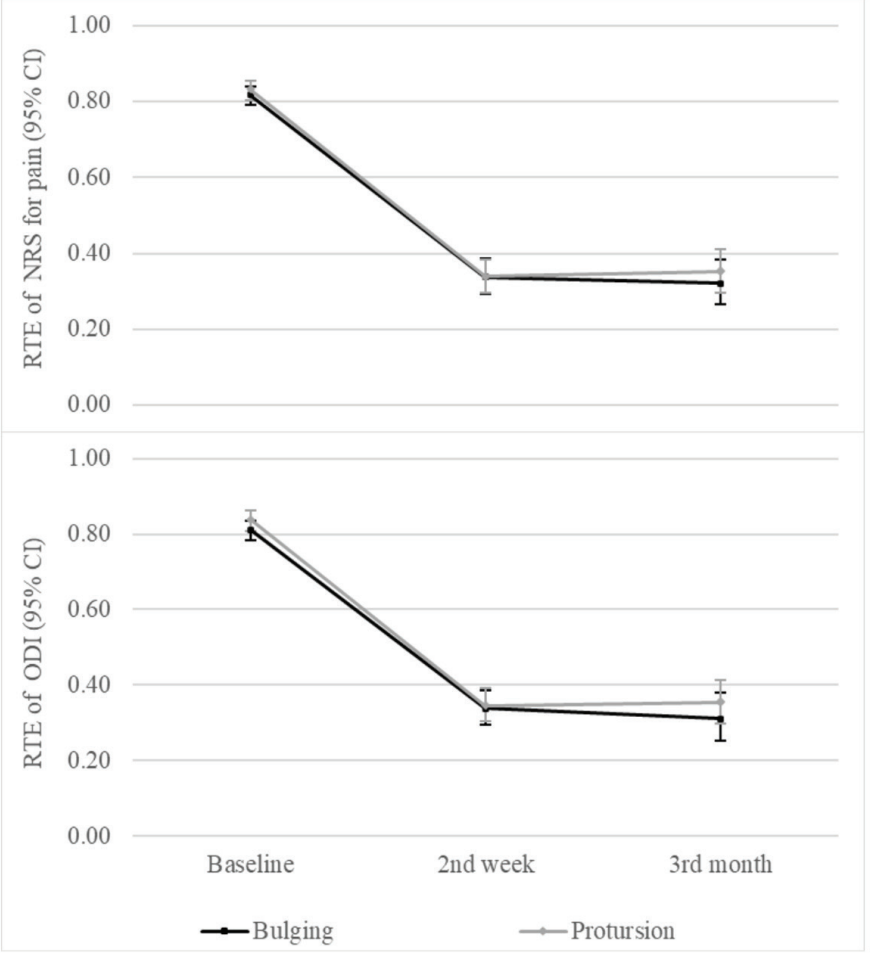

Figure 5. Relative treatment effect of pain and disability levels based on disc type

NRS: Numeric rating scale for pain, ODI: Oswestry disability index, RTE: Relative treatment effects, $\mathrm{Cl}$ : Confidence interval 
turkishspine

applied with local anesthetic and steroid in TFESI, moderate when only local anesthetic was applied; they found moderate evidence for radiculitis secondary to spinal stenosis with local anesthetics and steroids, and limited evidence for axial pain and postoperative syndrome using local anesthetics with or without steroids. We applied local anesthetics and steroids only to patients with LDH in our study, and we achieved significant improvement in patient groups regardless of disc types in MRI.

Although transforaminal epidural steroid injection (TFESI) is a useful diagnostic, prognostic and short-term therapeutic tool for lumbar radiculopathy, Leung et al. ${ }^{(13)}$ reported that it has been reported that although TFESI cannot change the need for surgery in the long term, it is a very safe procedure to provide short-term pain relief and as a preoperative evaluation tool. In our study, significant improvement was achieved both in the early and late periods compared to the pre-procedure. This improvement also confirms that it is due to the disc localization seen on MRI in a diagnostic sense.

To in epidural steroid administration methods; In terms of recovery and pain control in patients with unilateral lumbar radiculopathy; Makkar et al. ${ }^{(14)}$ reported that the transforaminal approach is equivalent to the parasagittal interlaminar application and superior to the midline interlaminar approach. Buenaventura et al. ${ }^{(15)}$ reported that epidural corticosteroid injection is one of the most commonly used interventions in the treatment of chronic spinal pain, the transforaminal pathway to

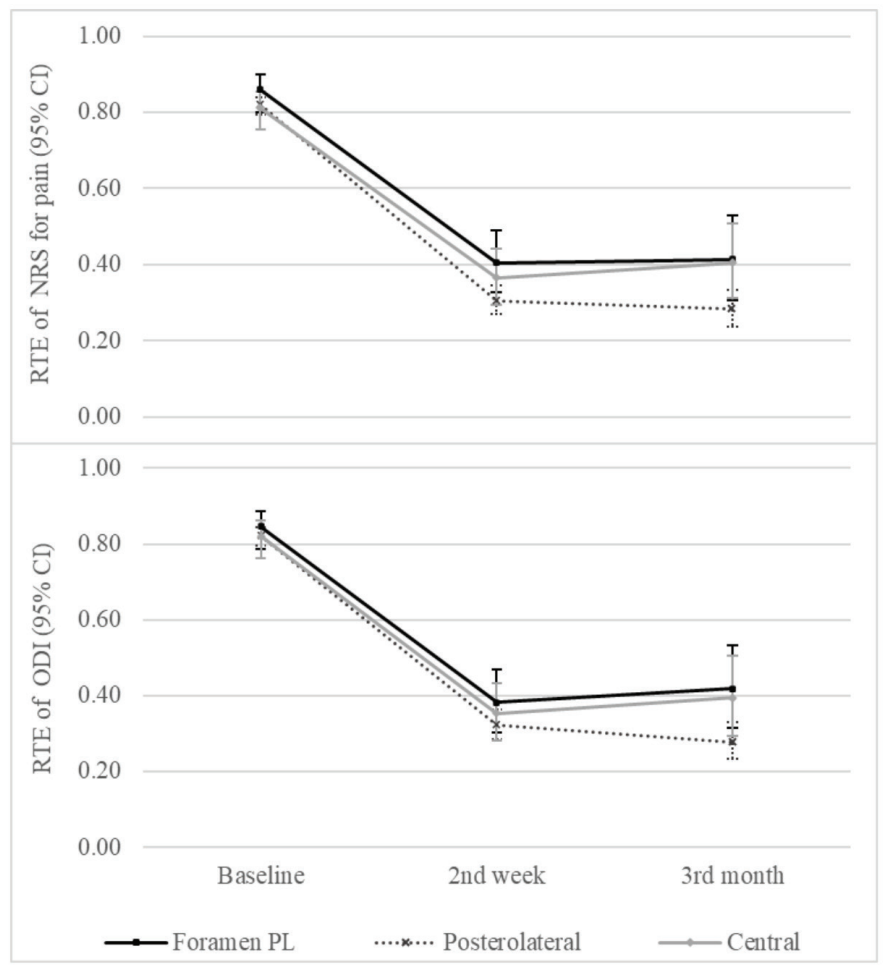

Figure 6. Relative treatment effect of pain and disability levels based on disc localization

NRS: Numeric rating scale for pain, ODI: Oswestry disability index, RTE: Relative treatment effects, $\mathrm{Cl}$ : Confidence interval the lumbar epidural space for steroid injection is a fast-acting and widely accepted method for the treatment of lumbar and leg pain. TFESI was applied to all patients in our study and successful results were obtained.

Tecer et al. ${ }^{(16)}$ have stated that TFESI is an effective treatment method in patients with radicular pain regardless of the type or location of disc herniation. Kwak et al.(17) in his study, there was no significant difference in TFESI results in patients with radiculopathy due to $\mathrm{LDH}$ according to the location, type and size of disc herniation determined on MRI. Parallel to this, in our study, no statistical correlation was observed between disc type and localization and VAS and ODI scores in the pre-procedural MRI.

Roberts et al.(18) found compelling evidence to support that TFESIs are superior to placebo in the treatment of radicular symptoms. They reported good evidence that TFESIs should be used as a prophylactic intervention and that TFESIs are superior to interlaminar and caudal epidural steroid injections for radicular pain ${ }^{(18)}$. Only TFESI method was applied to all of our patient group.

Kozlov et al. ${ }^{(19)}$ showed that epidural steroid and non-steroid injections are more effective than non-epidural injections in cases with radicular pain symptoms. In addition, studies have shown the effectiveness of non-particulate steroids to approximate the efficacy of particulate steroids. It supports the better efficacy of transforaminal injection due to the higher incidence of ventral epidural spread compared to interlaminar injection. Thus, they proposed a transforaminal approach when unilateral radicular pain is limited to a nerve root. However, the transforaminal approach is associated with a higher incidence of central nervous system injury, including paraplegia, which is attributed to particulate steroid embolization. Recent studies have shown that non-particulate steroids potentially last as long as particulate steroids. Therefore, they recommended the use of non-particle steroids in the first transforaminal epidural injection $^{(19)}$. Makkar et al. ${ }^{(20)}$ stated that the recovery scores of particulate steroids were slightly better than non-particle steroids, and stated that the clinician should weigh the risk of complications, however. We administered $5 \mathrm{cc}$ by mixing $1 \mathrm{cc}$ betamethasone and 4 cc $2 \%$ prilocaine hydrochloride to all our patients, and although a significant improvement was achieved in the patient groups, we did not encounter any complications. Although Roy et al. ${ }^{(21)}$ designed to inject the drug once, the longterm pain relief effect was found to be better in patients with pain duration less than 6 months, most of the patients needed a second injection and reported that better results could be obtained with multiple injections in a predetermined time interval. All of our patient group was a patient group that did not respond to conservative treatment for a minimal period of 1 month. In addition, the need for recurrent injections and cases leading to surgery, only single injection results were included in this study.

In this study, no correlation was found between the disc type and localization in MRI findings and the patients' response to 
treatment. There is a need for new studies with standardized MRI criteria and different injection practices, as well as larger study groups in different centers.

\section{CONCLUSION}

In patients with radiculopathy due to LDH and who do not benefit from conservative treatment; although it is not an alternative procedure to surgery, TFESI is a treatment method that can be used safely, regardless of the disc type and localization in the MRI taken before the procedure, considering its rapid effect in the early period and significant recovery results in the future.

\section{Ethics}

Ethics Committee Approval: Ethics committee approval was obtained for this study from Uludağ University Faculty of Medicine Clinical Research Ethics Committee (dated 16.06.2021 and numbered 2011-KAEK-26).

Informed Consent: Informed consent forms were obtained from all patients before the TFESI procedure.

\section{Authorship Contributions}

Surgical and Medical Practices: B.A., Concept: B.A., A.K., Design: B.A., A.K., Data Collection or Processing: B.A., A.K., Analysis or Interpretation: B.A., Literature Search: B.A., A.K., Writing: B.A., A.K.

Peer-review: Externally and internally peer-reviewed.

Financial Disclosure: The authors declared that this study received no financial support.

Conflict of Interest: The authors have no conflicts of interest to declare.

\section{REFERENCES}

1. Lechmann M, Rosskopf A, Ehrmann C, Sutter R, Pfirrmann CWA Peterson CK. Relationship of specific MRI findings to treatment outcomes in patients receiving transforaminal epidural steroid injections. Skeletal Radiol. 2016;45:1677-85.

2. Öcal Ö, Seçen AE, Divanlıoğlu D, Dağlar Z, Günerhan G, Fırat Oğuz E, et al. Evaluation Of Serum Thiol/Disulfide Homeostasis And IschemiaModified Albumin Levels In Lumbar Disc Herniation. J Turk Spinal Surg. 2021;32:139-43.

3. Manchikanti L, Cash KA, Pampati V, Falco FJE. Transforaminal epidural injections in chronic lumbar disc herniation: A randomized, doubleblind, active-control trial. Pain Physician 2014;17:489-501.

4. Rosenberg SK, Grabinsky A, Kooser C, Boswell MV. Effectiveness of transforaminal epidural steroid injections in low back pain: a one year experience. Pain Physician. 2002;5:266-70.
5. Carlos E. Rivera, MD. Lumbar Epidural Steroid Injections. Phys Med Rehabil Clin N Am. 2018;29:73-92.

6. Lechmann M, Rosskopf A, Ehrmann C, Sutter R, Pfirrmann CWA, Peterson CK. Relationship of specific MRI findings to treatment outcomes in patients receiving transforaminal epidural steroid injections. Skeletal Radiol. 2016;45:1677-85.

7. Ghahreman A, FRACS, Bogduk N. Predictors of a Favorable Response to Transforaminal Injection of Steroids in Patients with Lumbar Radicular Pain due to Disc Herniation. Pain Medicine 2011;12:871-9.

8. Mysliwiec LW, Cholewicki J, Winkelpleck MD, Eis GP. MSU classification for herniated lumbar discs on MRI: toward developing objective criteria for surgical selection. Eur Spine J. 2010;19:1087-93.

9. RStudio Team (2021). RStudio: Integrated Development Environment for R. RStudio, PBC, Boston, MA URL http://www.rstudio.com/.

10. Noguchi K, Gel YR, Brunner E, Konietschke F. nparLD: An R Software Package for the Nonparametric Analysis of Longitudinal Data in Factorial Experiments. J Stat Softw. 2021;50:1-23.

11. R Core Team (2021). R: A language and environment for statistical computing. R Foundation for Statistical Computing, Vienna, Austria.

12. Manchikanti L, Buenaventura RM, Manchikanti KN, Ruan X, Gupta $\mathrm{S}$, Smith HS, et al. Effectiveness of therapeutic lumbar transforaminal epidural steroid injections in managing lumbar spinal pain. Pain Physician. 2012;15:E199-245.

13. Leung SM, Chau WW, Hukuku GB, Mantar KY. Clinical value of transforaminal epidural steroid injection in lumbar radiculopathy. Hong Kong Med J. 2015;21:394-400.

14. Makkar JK, Gourav KKP, Jain K, Singh PM, Dhatt SS, Sachdeva N, et al. Transforaminal Versus Lateral Parasagittal Versus Midline Interlaminar Lumbar Epidural Steroid Injection for Management of Unilateral Radicular Lumbar Pain: A Randomized Double-Blind Trial. Pain Physician. 2019;22:561-73.

15. Buenaventura RM, Datta S, Abdi S, Smith HS. Systematic review of therapeutic lumbar transforaminal epidural steroid injections. Pain Physician. 2009;12:233-51.

16. Tecer D, Adiguzel E, Tan AK, Taskaynatan MA. Role of Magnetic Resonance Imaging in Ascertaining the Success of Transforaminal Epidural Steroid Injection for Lumbar Radicular Pain. Pain Med. 2017; 18:645-50.

17. Kwak S, Jang SH, Chang MC. Long-term outcomes of transforaminal epidural steroid injection in patients with lumbosacral radicular pain according to the location, type, and size of herniated lumbar disc. Pain Pract. 2021;21:836-42.

18. Roberts ST, Willick SE, Rho ME, Rittenberg JD. Efficacy of lumbosacral transforaminal epidural steroid injections: a systematic review. PM R. 2009;1:657-68.

19. Kozlov N, Benzon HT, Malik K. Epidural steroid injections: update on efficacy, safety, and newer medications for injection. Minerva Anestesiol. 2015;81:901-9.

20. Makkar JK, Singh PM, Jain D, Goudra B. Particulate vs non-particulate steroids for transforaminal epidural steroid injections: systematic review and meta-analysis of the current literature. Pain Physician. 2016;19:327-40.

21. Roy C, Chatterjee N, Patro SN, Chakraborty A, Vijay Kumar GR, Sengupta R. The efficacy of transforaminal epidural steroid injections in lumbosacral radiculopathy. Neurol India. 2011;59:685-9. 